\begin{tabular}{|c|c|}
\hline \multirow{3}{*}{ 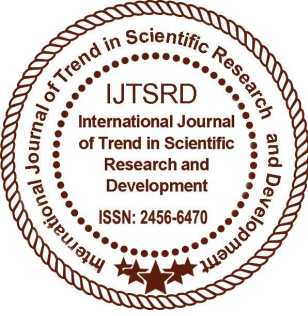 } & $\begin{array}{l}\text { International Journal of Trend in Scientific } \\
\text { Research and Development (IJTSRD) }\end{array}$ \\
\hline & International Open Access Journal \\
\hline & ISSN No: 2456 - 6470 | www.ijtsrd.com | Volume - 2 | Issue - 4 \\
\hline
\end{tabular}

\title{
Review on Linear Array Antenna with Minimum Side Lobe Level Using Genetic Algorithm
}

\author{
Aditya Sharma ${ }^{1}$, Er. Praveen Kumar Patidar ${ }^{2}$ \\ ${ }^{1} \mathrm{M}$. Tech (Student), ${ }^{2}$ Professor \\ Department of Electronics and Communication Engineering, Lakshmi Narain College of Technology, \\ Indore (Madhya Pradesh), India
}

\begin{abstract}
Antenna array is formed by assembly of radiating elements in an electrical or geometrical configuration. In most cases the elements are identical. In this paper proposed a very simple and powerful method for the synthesis of linear array antenna and GA. This method reduced the desired level of side lobe level (SLL) as well as to steer the main beam at differentdifferent angle. A new method for adaptive beam forming for a linear antenna arrays using genetic algorithm (GA) are also proposed.
\end{abstract}

Keywords: Genetic Algorithm (GA), Linear Arrays, Sidelobe Level (SLL), Polarization

\section{INTRODUCTION}

In design of antenna arrays, one of the most important parameter is sidelobe level (SLL). High side-lobes are undesirable as they result in Electro Magnetic Interference (EMI) which degrades the overall system performance [1], [2]. In wireless communication, one of the most recent inventions to overcome the problem of increasing demand for capacity is to deploy smart antenna [2-4]. Smart antennas have also adaptability to introduce new services, increased range, faster bit rate, multi use interference, space division multiplexing (SDMA), more security, reduction of errors due to multipath fading etc. Usage of the antenna arrays can improve the capacity and the spectral efficiency of a wireless communication system [3, 4]. For example, the fifth generation (5G) communications adopt the millimetre wave (mmwave) and beam forming technologies based on antenna arrays, to improve the spectral efficiency and communication rate of the system [5].

\section{A. Side-Lobes}

No antenna is able to radiate all the energy in one preferred direction. Some is inevitably radiated in other directions. The peaks are referred to as sidelobe, commonly specified in $\mathrm{dB}$ down from the main lobe, In figure 1 shows sidelobe and main lobe.

\section{B. Nulls}

In an antenna radiation pattern, a null is a zone in which the effective radiated power is at a minimum. A null often has a narrow directivity angle compared to that of the main beam. Thus, the null is useful for several purposes, such as suppression of interfering signals in a given direction.

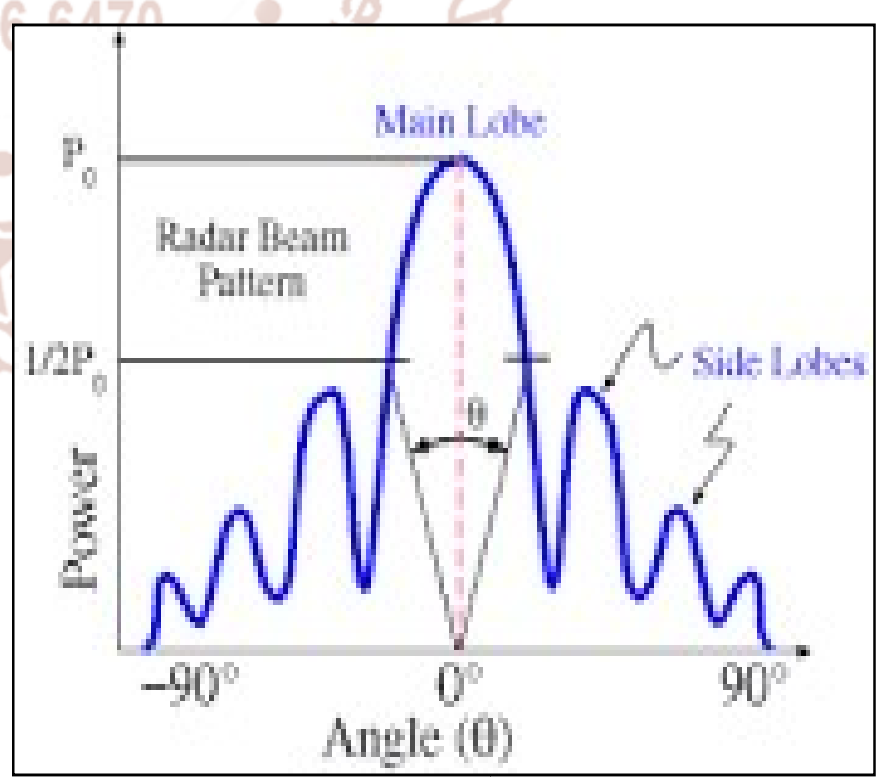




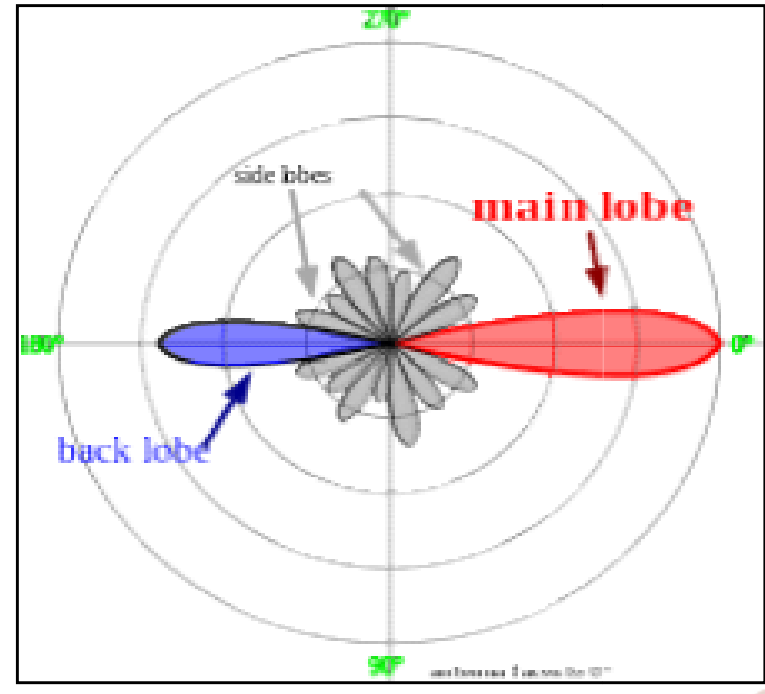

Fig 1: Side-Lobes, Main-Lobe and Back-Lobe.

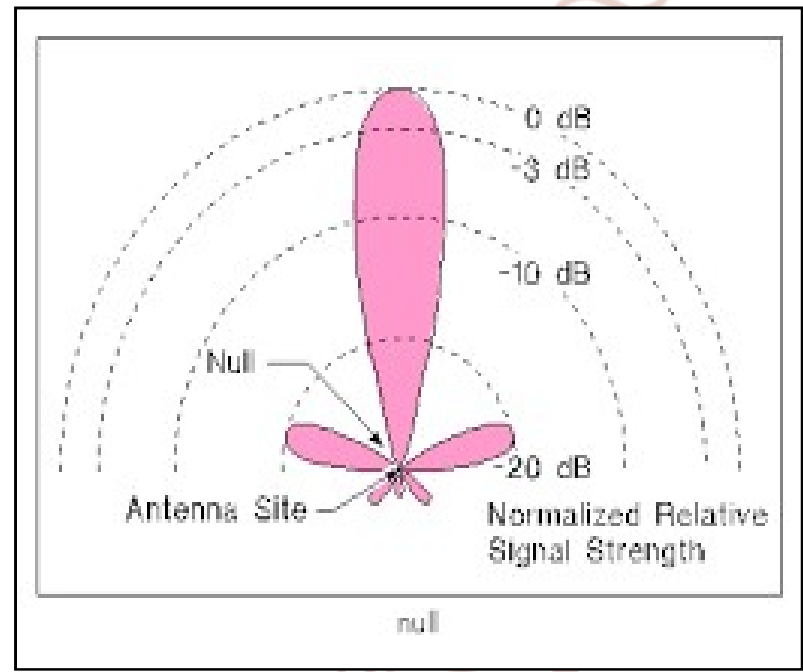

Fig. 2: Null antenna system show

\section{Polarization}

Polarization is defined as the orientation of the electric field of an electromagnetic wave. Polarization is in general described by an ellipse. Two special cases of elliptical polarization are linear polarization and circular polarization. The initial polarization of a radio wave is determined by the antenna. With linear polarization the electric field vector stays in the same plane all the time. Vertically polarized radiation is somewhat less affected by reflections over the transmission path. Omni directional antennas always have vertical polarization. With horizontal polarization, such reflections cause variations in received signal strength. Horizontal antennas are less likely to pick up man-made interference, which ordinarily is vertically polarized. In circular polarization the electric field vector appears to be rotating with circular motion about the direction of propagation, making one full turn for each RF cycle. This rotation may be right hand or left hand. Choice of polarization is one of the design choices available to the RF system designer.

\section{LITERATURE REVIEW}

1. Chuang Han and Ling Wang (2016), "Array Pattern Synthesis Using Particle Swarm Optimization with Dynamic Inertia Weight", Hindawi Publishing Corporation International Journal of Antennas and Propagation, 2016, In this paper (FPSO) with a family of fitness functions is proposed to minimize side lobe level (SLL) and control null. The optimized objectives in the fitness function can obtain an accurate null level independently. The directly constrained SLL range reveals the capability to reduce SLL. Considering both element positions and complex weight coefficients, a low-level SLL, accurate null at specific directions, and constrained main beam are achieved. Numerical examples using a uniform linear array of isotropic elements are simulated, which demonstrate the effectiveness of the proposed array pattern synthesis approach.

2. G. S. K. Gayatri Devi, G. S. N. Raju and P. V. Sridevi (2015), “ Application of Genetic Algorithm for Reduction of Sidelobes from Thinned Arrays"AMSE JOURNALS -2015, Vol. 58(1), This paper presents the design of linear array of isotropic elements which generates low sidelobe patterns. The array is excited with prefixed amplitude taper and simultaneously thinned. A Genetic Algorithm is employed to find the optimum thinning configurations. Numerically simulated results are presented for different number of elements.

3. Rajesh Beraa and Ragini Lanjewar (2015) "Comparative Study of Circular and Hexagonal Antenna Array Synthesis using Improved Particle Swarm Optimization" Elsevier, Procedia Computer Science Vol. 45. This paper describes the comparison of the performance of Circular array (CA) and Hexagonal Array (HA) of uniformly excited isotropic antennas which can generate directive beam with minimum relative Side Lobe Level (SLL). This paper is basically concerned with the comparison of the performance of thinned $\mathrm{CA}$ and $\mathrm{HA}$ in terms of SLL by fixing the other array design variable.

4. Smita Banerjee, Ved Vyas Dwivedi et al. (2015), "Linear Antenna Array Synthesis to Reduce the Interference in the Side Lobe using 
Continuous Genetic Algorithm” IEEE-2015 Fifth International Conference on Advances in Computing and Communications, This paper discusses the problem of interference suppression by reducing the side lobe. Synthesis of linear antenna array using Continuous Genetic Algorithm is done to search for the optimum amplitude weights to minimize the maximum side lobe level. It has been demonstrated that side lobe level is reduced effectively by this technique. The simulated results show an improved directivity, reduction in the first side lobe and fast convergence. This shows the effectiveness of this technique.

5. Shishir Kumar Singh et. al. (2013) proposed their paper and he give a very simple and powerful method for the optimization of linear array antenna. This method obtained the desired level of side lobe level (SLL) along with the minimum desired value of first null beam width (FNBW). These Method includes finding a set of non-uniform amplitude excitation using particle swarm optimization (PSO) and finding the appropriate value of element spacing for the linear antenna array, which produces the desired value of SLL and provides minimum desired FNBW value. This method finds its application where the max SLL criterion for the radiation pattern is fixed . the simulation are performed on MATLAB, The numbers of elements taken for the simulation are 20, 26 and 30 and the SLL obtained -40.002, $40.074,-40.008$ and corresponding FNBW is obtained 15-69,12.08, 7.780 in degree.

6. Bipul Goswami, Durbadal Mandal, (2012) "Genetic algorithm for nulls and side lobe level control in a linear antenna array", Production and hosting by Elsevier, King Saud University Journal of King Saud University -Computer and Information Sciences. In this paper Real coded Genetic Algorithm (RGA) is used to determine an optimal set of current excitation weights of antenna elements and optimum inter-element spacing that satisfy the optimal goal.

7. Slawomir Koziel et. al., (2012), presented their paper on End-Fire Array Synthesis Using Gradient-Based Numerical Optimization with Analytical Derivatives. In this paper numerical optimization has been investigated. Analytical derivatives of the array directivity with respect to the designable variables and optional initialization through a smart random search have been utilized. The SLL has been reduced at a low computational cost without compromising the directivity compared to the HW design and without shrinking the spacing. The optimization results for SLL reduction with uniform spacing and uniform phase shift is $-12.97,-13.19,-13.25$ with antenna element of 10, 20, 40. Optimization Results For Maximum Directivity With Uniform Spacing And Uniform Phase Shift is $-10.50,-10.68,-10.50$ with respective directivity.

8. D. Mandal, A. K. Bhattacharjee et. al. (2011), In this paper the synthesis of linear array geometry with minimum side lobe level using Improved Particle Swarm Optimization (IPSO) is described. The IPSO algorithm is a newly proposed, highperformance evolutionary algorithm which is capable of solving general $\mathrm{N}$-dimensional, linear and nonlinear optimization problems. Compared to other evolutionary methods such as genetic algorithms and simulated annealing, the IPSO algorithm is much easier to understand and it implemented easily and requires the least of mathematical calculation. This section gives the simulated results for various linear array designs obtained by IPSO technique. The set of linear array designs considered are 12, 16, 20 and 24 . From the comparison, it can be observed that the fitness function $(M F)$ along with the IPSO method gives better radiation pattern than that obtained from uniformly excited ( $I m=1)$ linear array with spacing of $\lambda / 2$ between elements, although the number of elements are the same. The SLL reduced to $-13.14 \mathrm{~dB}$ and $-13.21 \mathrm{~dB}$ SLL IPSO with optimal non-uniform current excitation and inter element spacing provide maximum SLL reductions to $-42.7 \mathrm{~dB},-43.5 \mathrm{~dB}$ respectively. The programming has been written in Matlab language using MATLAB 7.5 version on core (TM) 2 duo processor, $3.00 \mathrm{GHz}$ with $2 \mathrm{~GB}$ RAM.

9. Khyati R.Zalawadia et. al. (2011), "Adaptive Beam Former Design using RLS Algorithm for Smart Antenna System" This paper focuses on adaptive beam forming approach used in smart antennas and Recursive Least Square (RLS) adaptive algorithm used to compute the complex weights by own simulation. The performance of the RLS algorithm is analyzed, in the presence of Additive White Gaussian Noise (AWGN) and two 
interferences. Our all results and graphs are simulated using MATLAB software. This problem is solved with the RLS algorithm by replacing the gradient step size _ with a gain matrix. It was noticed that increasing the number of elements of the antenna array ensures better performance. Also conclude that the optimum spacing beam between the elements is half wave length.

\section{SMART ANTENNA CONSTRUCTION}

Omni-directional or sectored antennas used in current wireless communication systems, can be considered as an inefficient use of power as most of it has been radiated in other directions than toward the user. Signals that miss the intended user will cause interference to other users in the same or adjoining cells [1]. The concept of smart antennas is to employ base station antenna patterns that are not fixed in any direction but adapt to the current radio conditions. In other words, the antenna is to direct a single beam to each user. Smart antennas direct their main lobe, with increased gain, in the direction of the user, and they direct nulls in directions away from the main lobe [23].

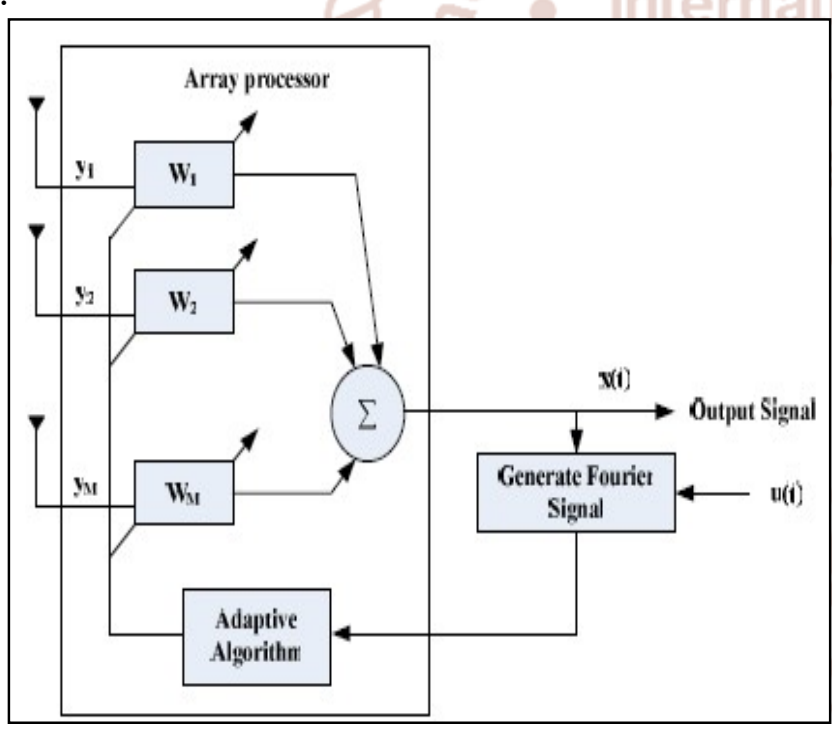

Fig. 3: Consist of an array of antenna elements

Shown in Figure 3, consist of an array of antenna elements and a smart processing of antenna signals. We will concentrate on the adaptive arrays that make use of the Direction of Arrival (DOA) information from the desired user to steer the main beam towards the desired user. The signals received by each antenna element are weighted and combined to create a beam in the direction of the mobile by utilizing signal processing signal processing algorithms [4]. These algorithms determine the uplink weight vectors for performing beam-forming on the received signals as well as the downlink weight vectors for performing beam forming on the transmitted signals [3].

\section{GENETIC ALGORITHM}

Genetic Algorithm is an evolutionary algorithm developed on the principles of genetics [5]. It is a nature inspired algorithm [6]. A genetic algorithm is a search technique used in computing to find exact or approximate solutions to optimization and search problems.

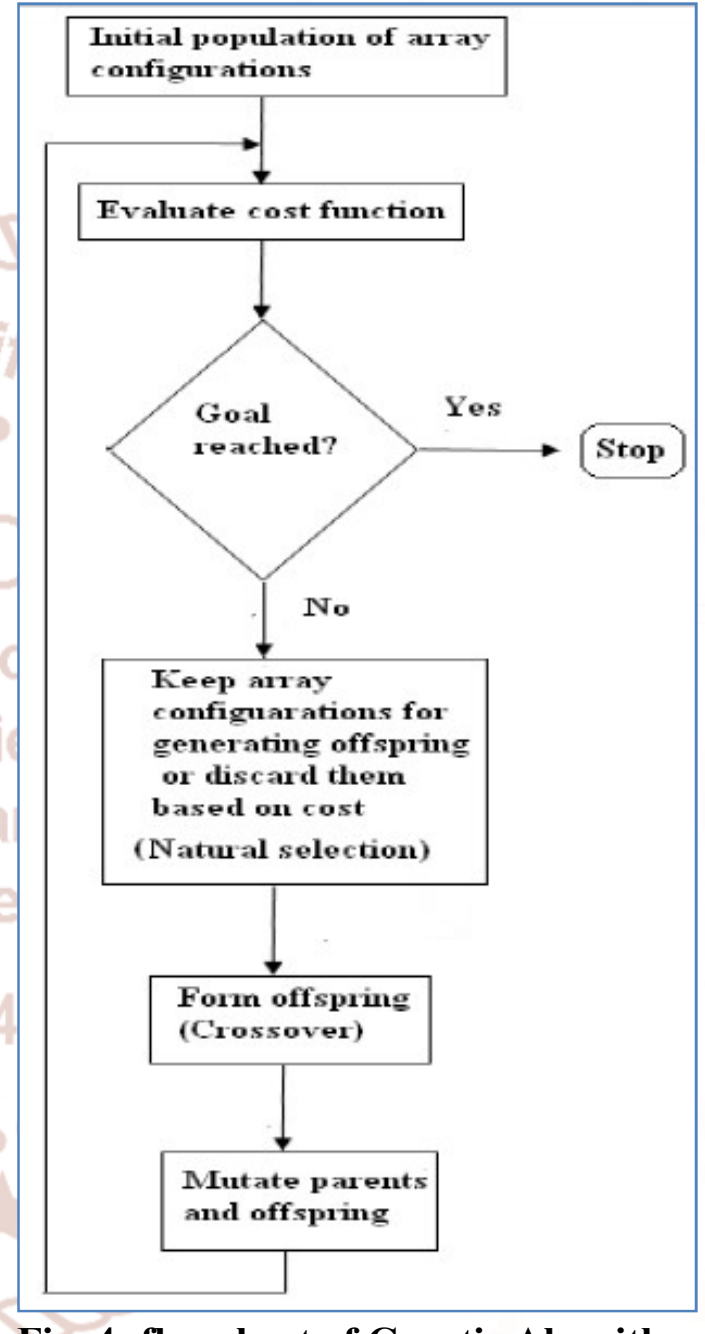

Fig. 4: flowchart of Genetic Algorithm

Genetic algorithms are a particular class of evolutionary algorithms (also known as evolutionary computation) that use techniques inspired by evolutionary biology such as inheritance, mutation, selection, and crossover (also called recombination). Genetic algorithms are search algorithms based on mechanics of natural selection and natural genetics. In every generation, a new set of artificial creatures or strings is created using bits and pieces of the fittest of the old.

In order to use antenna placement on crowded platforms, there are three methods for synthesizing amplitude and phased array excitations on uniformly 
arranged antenna element were computer programmed and compared: the numerical method, the analytic method and the Genetic Algorithm method (GA).

The one of the most important parameters in array designing is side lobe level (SLL) and first null beam width (FNBW). In array antenna, the desired value of parameter can be achieved by number of ways such as by having variation in the geometry configuration of antenna, variation in current amplitude or phase feed to the antenna elements. A flowchart of Genetic Algorithm is shown in fig.4. The steps required for implementing the algorithm are as follows: 1 . Define the fitness function, select parameters to be optimized by GA, 2. Generate initial population, 3. Calculate fitness, 4. Selection, 5. Crossover, 6. Mutation, 7. Check for stopping criteria, stop if it is satisfied, 8. Go to step 3 .

\section{SIMULATION BLOCK DIAGRAM}

The simulation block diagram for optimization of linear array antenna using GA for reduction in Side Lobs Levels, as shown in figure 5 .

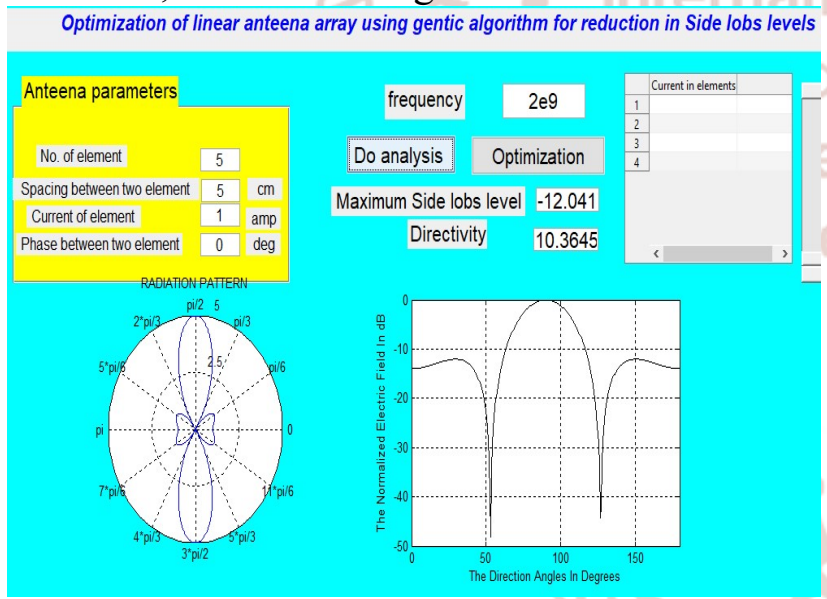

\section{CONCLUSION}

Genetic Algorithms are adaptive heuristic search algorithm based on the evolutionary ideas of natural selection and genetics. As such they represent an intelligent exploitation of a random search used to solve optimization problems. Antenna pattern synthesis is an important topic in the smart antenna. This is the process of choosing various antenna parameter to obtain the given radiation pattern of antenna array like beam width, specific position of null, side lobe level etc. smart antenna which have great interest in many scientific fields such as telecommunication, medicine, military and astronomy thank to their precision.

\section{REFERENCES}

1. G. S. N. Raju (2005), "Antennas and wave propagation," Pearson Education.

2. Rao. P. M, Sridevi. P. V, Gutti. C. S, Prasad. M. V. V, Raju. G. S. N, Raju. K. V. L (6-8th December 1995), "Some Studies on Arrays for Sidelobe Reduction required for EMI Control," International Conference on Electromagnetic Interference and Compatibility, Madras, Madras, pp. 314-320, IEEE.

3. S. Liang, T. Feng, and G. Sun, "Sidelobe-level suppression for linear and circular antenna arrays via the cuckoo search chicken swarm optimisation algorithm," IET Microwaves, Antennas \& Propagation, vol. 11, no. 2, pp. 209-218, 2016.

4. I. F. Akyildiz, S. Nie, S. C. Lin, and M. Chandrasekaran, "5G roadmap: 10 key enabling technologies," Computer Networks, vol. 106, pp. 17-48, 2016.

5. D. Z. Zhu, P. L. Werner, and D. H. Werner, "Design and optimization of 3-D frequencyselective surfaces based on a multi objective lazy ant colony optimization algorithm," IEEE Transactions on Antennas \& Propagation, vol. 65, no. 12, pp. 7137-7149, 2017.

6. G. S. N. Raju, G. M. V. Prasad (2003), " Some investigations on the generation of sum and difference patterns from array antennas," AMSE Journal, Advances A, France, vol.40, no.1, pp.3140.

7. - Chuang Han and LingWang (2016), "Array Pattern Synthesis Using Particle Swarm Optimization with Dynamic Inertia Weight", Hindawi Publishing Corporation International Journal of Antennas and Propagation Volume 2016, pp. 1- 7.

8. G. S. K. Gayatri Devi, G.S.N.Raju, P.V.Sridevi (2015), " Application of Genetic Algorithm for Reduction of Sidelobes from Thinned Arrays" AMSE JOURNALS -2015-Series: Advances B; Vol. 58; $N^{\circ} 1$; pp 35-52, 2015

9. Smita Banerjee, Ved Vyas Dwivedi et al. (2015), "Linear Antenna Array Synthesis to Reduce the Interference in the Side Lobe using Continuous Genetic Algorithm" IEEE-2015

10. Bipul Goswami, Durbadal Mandal "Genetic algorithm for nulls and side lobe level control in a linear antenna array" (2012), Production and hosting by Elsevier, King Saud University Journal of King Saud University -Computer and Information Sciences. 